\title{
Spiral Bacterial Cell Image Analysis using Active Contour Method
}

\author{
P.S. Hiremath \\ Department of Computer Science, Gulbarga \\ University, Gulbarga, Karnataka, India
}

\author{
Parashuram Bannigidad \\ Dept. of Computer Science, \\ Govt. College, Gulbarga-585 105, Karnataka, India
}

\begin{abstract}
Image segmentation is a fundamental task in image analysis responsible for partitioning an image into multiple subregions based on a desired feature. Active contours have been widely used as attractive image segmentation methods because they always produce sub-regions with continuous boundaries, while the kernel-based edge detection methods, e.g. Sobel edge detectors, often produce discontinuous boundaries. The objective of the present study is to develop an automatic tool to identify and classify the different types of spiral bacterial cells in digital microscopic cell images using active contour method. Geometric features are used to identify the arrangement of spiral bacterial cells, namely, vibrio, spirillum and spirochete. The current methods rely on the subjective reading of profiles by a human expert based on the various manual staining methods. In this paper, we propose a method for bacterial classification by segmenting digital spiral bacterial cell images and extracting only three geometric features for cell classification using different classifiers, namely, $3 \sigma$ classifier, K-NN classifier, Neural Network classifier and Neuro Fuzzy classifiers. The experimental results are compared with the manual results obtained by the microbiology expert and demonstrate the efficacy of the proposed method.
\end{abstract}

\section{Keywords}

Cell segmentation; bacterial image analysis; vibrio; spirillum, spirochete; $3 \sigma$ classifier; K-NN classifier; Neural Network classifier; Neuro Fuzzy classifier; Active Contour Method.

\section{INTRODUCTION}

Automated microscopic image analysis provides an efficient tool for qualitative analysis in modern bio-medical and biological studies. Microscopic image analysis is one of the most important techniques in microbial ecology, since this is the most direct approach to examine the microbe's world from its own perspective. The value of quantitative microscopy in studies of microbial ecology can be increased even further when used in conjunction with computer-assisted image analysis. The main advantages of using digital image processing and pattern recognition techniques in conjunction with microscopy for quantitative studies of microbial ecology are; automatic image analysis reduces the amount of tedious work with microscopes needed to perform a more accurate quantitative analysis and these techniques provide an important quantitative tool to analyze the structures and spatial features of complex microbial communities. The major types of information useful in microbial ecology can be extracted from segmented microscopy images are; recognition of cellular morphological diversity, cell abundance, and spatial, metabolic, and phylogenetic relationships of cells to each other and their surrounding environment. The process of semi-automatic image analysis of cells to evaluate these aspects of microbial communities are; image acquisition, digitization, and segmentation; automatic measurement to extract features of interest; classification of different bacterial cells; and statistical analysis, computations, and interpretation of the data. One of the most important and yet most tedious tasks performed during microscopic analysis of microbial communities is the classification of observed cells into known morphological categories and recognition of new categories as well, if new distinct characteristics are captured [7].

\subsection{Arrangement of Spiral bacterial cell groups}

Spirals come in one of three groups: namely, vibrio, spirillum, and spirochete. Vibrio is a curve-shaped bacterium that looks like a "comma" or a "curl". Spirillum is a thick and a rigid spiral. Spirillum appears similar as the spirochete but its long axis remains rigid when in motion. Spirochete is a thin, flexible spiral. The Spirochete has long axis that bends when in motion. Spirals range in size from $1 \mu \mathrm{m}$ to over 100 $\mu \mathrm{m}$ in length [8]. The arrangement of spiral bacterial cell groups is shown in the Fig. 1 .

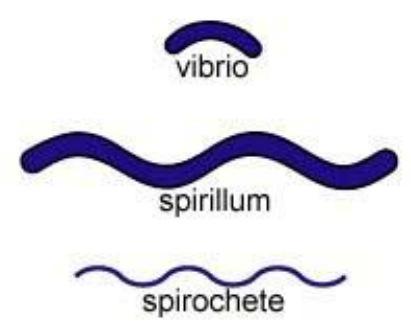

Fig. 1 Arrangement of Spiral bacterial cell groups

Many research works have been published in this context, the statistical imaging method for automatic identification of bacterial types is proposed by Trattner and Greenspan [13]. The artificial neural network approach for bacterial classification has been investigated by Nicholas Blackburn, et al. [9]. The data mining techniques are employed for the classification of HEp-2 cells by Petra Perner [11], in which a simple set of shape features are used for classification of bacterial cells. Hiremath and Parashuram [4, 9] have investigated the automatic classification of cocci and spiral bacterial cells and its sub groups using digital microscopic images using geometric shape features. A computer-aided system for the image analysis of bacterial morphotypes in microbial communities using geometric shape features has been investigated by J. Liu et al. [7]. Thomas Posch et al. [14] have proposed a new image analysis tool to study biomass and morphotypes of three major bacterioplankton groups in an alpine lake using geometric features. Carolina Wählby et al. [2] have investigated algorithms for cytoplasm 
segmentation of fluorescence labeled cells using statistical analysis techniques based on shape descriptive features. Efficient automated method for image-based classification of microbial cells has been investigated by Pekka Ruusuvuori et al. [16].

Digital microscopic image analysis of spiral bacterial cell groups using segmentation based on thresholding, feature extraction by leading to $100 \%$ classification accuracy by using five geometric features and neural network and fuzzy classifiers [19]. In the present paper, the objectives is to enhance the segmentation accuracy by using active contour method and reduced the complexity by using only three geometric features and yet attain 100\% classification accuracy by neural network and neuro fuzzy classifiers. The experimental results are compared with the manual results obtained by microbiology expert and demonstrate the efficacy of the proposed method.

\section{MATERIALS AND METHODS}

The spread plate technique is used for the separation of a dilute mixed population of micro-organisms, so that individual colonies can be isolated. Aseptically transfer the scoopful of mixed culture on the Nutrient Agar medium. Spread uniformly with the help of L-shaped spreader on the surface of medium plates. After spreading, incubate at $37^{\circ} \mathrm{C}$ for 24-48 hours. After incubation, single colonies will appear on the Nutrient Agar media plates. Then pick up the colony and go further identification by using staining techniques. A smear of mixed culture bacteria is deposited on a glass slide and thoroughly air-dried. It is stained for $1 \mathrm{~min}$ in Crystal Violet solution, $1 \mathrm{~min}$ in iodine solution, washed for $20 \mathrm{~s}$ in ethanol and finally, counterstained with Safranin for $1 \mathrm{~min}$. The glass slide is examined under oil immersion at $1000 \mathrm{x}-$ 2500x magnification with direct illumination in a Dialux 20 microscope equipped with a 3 CCD Sony color camera and connected to a PC $[1,9]$. We have considered 100 color TEM images for present study and these are converted into gray scale images [3].

\section{PROPOSED METHOD}

Image segmentation is a fundamental task in image analysis responsible for partitioning an image into multiple subregions based on a desired feature. Active contours have been widely used as attractive image segmentation methods because they always produce sub-regions with continuous boundaries, while the kernel-based edge detection methods, e.g. Sobel edge detectors, often produce discontinuous boundaries. Snakes or active contours, are curves defined within an image domain that can move under the influence of internal forces coming from within the curve itself and external forces computed from the image data. The internal and external forces are defined so that the snake will conform to an object boundary or other desired features within an image. Snakes are widely used in many applications, including edge detection, shape modelling, segmentation, and motion tracking.

The basic idea in active contour models or snakes is to evolve a curve, subject to constraints from a given image $u_{0}$, in order to detect objects in that image. For instance, starting with a curve around the object to be detected, the curve moves towards its interior normal and has to stop on the boundary of the object.
Let $\Omega$ be a bounded open subset of $\mathrm{R}^{2}$, with $\partial \Omega$ its boundary. Let $u_{0}: \bar{\Omega} \rightarrow \mathrm{R}$ be a given image, and $\mathrm{C}(s):[0,1] \rightarrow \mathrm{R}^{2}$ be a parameterized curve.

In the classical snakes and active contour models [5, 6, 20], an edge-detector is used, depending on the gradient of the image $u_{0}$, to stop the evolving curve on the boundary of the desired object. The snake model is: $\inf _{\mathrm{C}} \mathrm{J}_{1}(C)$, where

$$
\begin{gathered}
\mathrm{J}_{1}(C)=\alpha \int_{0}^{1}\left|\mathrm{C}^{\prime}(s)\right|^{2} d \mathrm{~s}+\beta \int_{0}^{1}\left|\mathrm{C}^{\prime \prime}(s)\right|^{2} d \mathrm{~s} \quad \ldots(1) \\
-\lambda \int_{0}^{1}\left|\nabla u_{0}(\mathrm{C}(\mathrm{s}))\right|^{2} d \mathrm{~s}
\end{gathered}
$$

Here $\alpha, \beta$ and $\lambda$ are positive parameters. The first two terms control the smoothness of the contour(the internal energy), while the third term attracts the contour toward the object in the image (the external energy). Observe that, by minimizing the energy (1), we are trying to locate the curve at the points of maxima $\left|\nabla u_{0}\right|$, acting as an edge-detector, while keeping a smoothness in the curve (object boundary).

A general edge-detector can be defined by a positive and decreasing function $g$, depending on the gradient of the image $u_{0}$, such that

$$
\lim _{z \rightarrow \infty} g(z)=0
$$

For instance,

$$
g\left(\left|\nabla u_{0}(x, y)\right|=\frac{1}{1+\left|\nabla G_{\sigma}(x, y) * u_{0}(x, y)\right|^{p}}, \mathrm{p} \geq 1\right.
$$

where $G_{\sigma} * u_{0}$, a smoother version of $u_{0}$, is the convolution of the image $u_{0}$ with the Gaussian $G_{\sigma}(\mathrm{x}, \mathrm{y})=\sigma^{-1 / 2} e^{-\left|x^{2}+y^{2}\right| / 4 \sigma}$. The function $g\left(\left|\nabla u_{0}\right|\right)$ is positive in homogeneous regions, and zero at the edges.

The purpose of the automated image analysis of digital bacterial cell image is to identify the type of bacteria whether it is vibrio, spirillum and spirochete based on their geometric features using different classification techniques, namely, $3 \sigma$ classifier, K-NN classifier, Neural Network classifier and Neuro Fuzzy classifiers.

The purpose of the automated image analysis of digital bacterial cell image is to identify the type of bacteria whether it is vibrio, spirillum and spirochete based on their geometric features using different classification techniques, namely, $3 \sigma$ classifier, K-NN classifier, Neural Network classifier and Neuro Fuzzy classifiers.

$\begin{array}{ll}\text { Circularity }\left(x_{1}\right): & \begin{array}{l}4 \pi(\text { Area }) / \text { perimeter }^{2} \\ \text { Eccentricity }\left(x_{2}\right):\end{array} \\ \begin{array}{l}\text { It is the ratio of the length of the highest } \\ \text { chord of the shape to the longest chord } \\ \text { perpendicular to it; i.e. }\end{array} \\ \begin{array}{l}\text { Length } \\ \text { major_axis/Length }\end{array}\end{array}$

\subsection{Classification rules}

\subsection{1 $3 \sigma$ Classifier}

The color spiral bacterial cell image is converted into gray scale image and adjusts the image intensity values. Perform active contour without edges upto 1600 iterations to obtain segmented image, which yields binary image. After labeling the segmented image, the geometric features $x_{i}, i=1,2,3$ are extracted for each labeled segment. These features are used as a basis for the cell classification. Using the training set of images (with known cell classification), for each feature $x_{i}^{k}, i=1,2,3$ of $\mathrm{k}^{\text {th }}$ cell type, we compute the mean $\bar{x}_{i}^{k}$ and 
standard deviation $\sigma_{i}^{k}$ of the sampling distribution of the feature values and store them as knowledge base. In the testing phase, for a given test image, feature values $x_{i}^{(\text {test })}$ of the segmented regions (cells) are computed and then cell classification is done using the $3 \sigma$ rule, namely: For a segmented region in the test image, if the feature values $x_{i}^{\text {(test) }}$ lie in the interval $\bar{x}_{i}^{k} \pm 3 \sigma_{i}^{k}, i=1,2,3$ then the region is a cell of type $\mathrm{k}$. The $\mathrm{k}=1,2,3$ correspond to vibrio, spirillum and spirochete, respectively.

\subsection{2. $\mathrm{K}$-NN Classifier}

The K-nearest neighbor (K-NN) classification is performed by using a reference data set (training set) which contains both the input (feature set) and the target variables (known cells) and then by comparing the unknown (test data) which contains only the input variables (features) to that reference set. The distance of the unknown to the $\mathrm{K}$ nearest neighbors determines its class assignment by either averaging the class numbers of the $\mathrm{K}$ nearest reference points or by obtaining a majority vote from them.

\subsubsection{Neural Network Classifier}

The input layer has 3 neurons and 3 shape features as inputs, and output layer has three outputs (vibrio, spirillum and spirochete). The transfer function used is 'tan sigmoidal', training function used is Levenberg-Marquardt back propagation, the weight/bias learning function is 'gradiant descent' function and the performance function is 'mean square error (mse)' which is set to 0.01 . In the case of radial basis neural network, the shape features are used as inputs. The error function is 'mean square error (mse)' which is set to 0.15 . The spread for radial basis function is 1.0 and the maximum number of neurons allowed to add during training is 300 [10].

\subsubsection{Adaptive Neuro-Fuzzy Inference System (ANFIS)}

ANFIS is one of hybrid neuro-fuzzy inference expert systems and it works as Takagi-Sugeno type fuzzy inference system. ANFIS has a similar structure to a multilayer feed forward neural network, but the links in an ANFIS only indicate the flow direction of signals between nodes and no weights are associated with the links. The ANFIS uses a strategy of hybrid approach of adaptive neuro-fuzzy inference and yields good classification results [17]. The objective of classification is to classify three types of spiral bacterial cells. The feature vectors were applied as the input to an ANFIS classifier. The ANFIS network has a total of 81 fuzzy rules and one output. The algorithm for classification of spiral bacterial cell image by ANFIS is given in the Algorithm 2 and is implemented using MATLAB.

The algorithm of the proposed method for the classification of spiral bacterial cell groups based on their geometric features is given in section 3.2.

\subsection{Training and Testing algorithms}

\subsubsection{Training phase:}

Algorithm 1: Extraction of features for knowledge base

Step 1: Input bacterial cell image (RGB color training image).

Step 2:Convert the RGB image into gray scale image And adjust the image intensity values.

Step 3: Perform active contour without edges upto 1600 iterations to obtain segmented image.

Step 4: Binarize the segmented image of step 3.
Step 5: After removing border touching cells, perform labeling the segmented image.

Step 6: For each labeled segment, compute geometric shape features $x_{i}, i=1,2,3$ (i.e. eccentricity, circularity and tortuosity) for each cell type $\mathrm{k}$. The $\mathrm{k}=1,2,3$ correspond to vibrio, spirillum and spirochete, respectively.

Step 7: Repeat steps 1 to 5 for all the training images.

Step 8: Compute mean $\bar{x}_{i}^{k}$ and standard deviation $\sigma_{i}^{k}$ of the sampling distribution of the feature values for each cell type $\mathrm{k}$ and store them as knowledge base.

3.2.2. Classification phase:

Algorithm 2: Classification of bacterial cells.

Step 1: Input bacterial cell image (RGB color test image).

Step 2: Convert the RGB image into gray scale image and adjust the image intensity values.

Step 3: Perform active contour without edges upto 1600 iterations to obtain segmented image.

Step 4: Binarize the segmented image of step 3.

Step 5: After removing border touching cells, perform labeling the segmented image.

Step 6: For each labeled segment, compute geometric shape features $x_{i}, i=1,2,3$ (i.e. eccentricity, circularity and tortuosity) and store these features as $x_{i}^{(t e s t)}$.

Step 7: Apply $3 \sigma$ rule for classification of the bacterial cells: A segmented region is of cell type $\mathrm{k}$, if its features $x_{i}^{(\text {test })}$ lie in the interval $\bar{x}_{i}^{k} \pm 3 \sigma_{i}^{k}, i=1,2,3$ The $\mathrm{k}=1,2,3$ correspond to vibrio, spirillum and spirochete, respectively.

Step 8: Repeat the Steps 6 and 7 for all labeled segments and output the classification of identified cells.

The above algorithm for classification phase can be modified to apply K-NN classifier, Neural Network classifier and Neuro Fuzzy classifier to the feature set in the Step 7 and the classification performance of the different classifiers can be compared. The $\mathrm{K}-\mathrm{NN}$ classifier with $\mathrm{K}=1$ is the minimum distance classifier.

\section{EXPERIMENTAL RESULTS AND DISCUSSIONS}

For the purpose of experimentation, 300 color digital bacterial cell images containing different types of bacterial cells (nonoverlapping) namely, vibrio, spirillum and spirochete are considered (as described in section 2). The implementation is done on a Pentium Core 2 Duo @ $2.83 \mathrm{GHz}$ machine using MATLAB 7.9. In the training phase, each input color image of bacterial cell (Fig. 2(a)) is converted into gray scale image and adjusts the image intensity values using MATLAB function. The resultant image is initiated by a fixed rectangular mask (Fig. 2 (b)), and then, segmented using active contour method (Fig. 2(c)) to obtain binary image (Fig. (d)). Then, the segmented image is labeled and for each segmented region (known cells), the geometric shape features are computed. The Table 1 presents the geometric feature values computed for the segmented cell regions of the image in the Fig. 1(d).

The mean and standard deviation of the sampling distribution of these features obtained from the training images are stored in the knowledge base of the cells: Vibrio, Spirillum and Spirochete, as shown in Table 2. Sample training images of spiral bacterial cells are shown in Fig. 3 . 
(a)

1600 Iterations

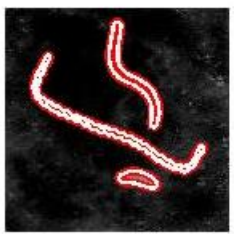

(c)

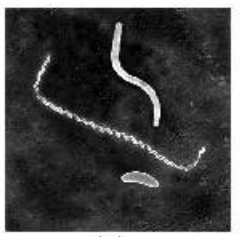

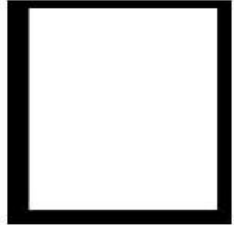

(b)

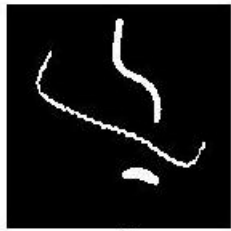

(d)
Fig. 2. (a) Original Spiral color cell image, (b) Initializing fixed rectangular grid image of color image in (a), (c) image after 1600 iterations, (d) Binarized image after segmentation by active contour method.
Table 1. The geometric feature values of the cell regions of the image in Figure. 2(d)

\begin{tabular}{|c|l|l|l|}
\hline Cell & Vibrio & Spirillum & Spirochete \\
\hline Circularity $\left(\mathrm{x}_{1}\right)$ & 0.4294 & 0.1103 & 0.0265 \\
\hline Eccentricity $\left(\mathrm{x}_{3}\right)$ & 0.9444 & 0.9768 & 0.9865 \\
\hline Tortuosity $\left(\mathrm{x}_{4}\right)$ & 0.3779 & 0.3970 & 0.3647 \\
\hline
\end{tabular}
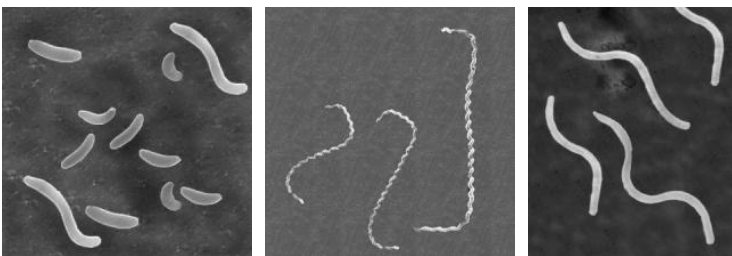

Fig. 3. Sample training images of spiral bacterial cells

In the testing phase, for a test image, the feature extraction algorithm is applied and the test feature values $x_{i}^{(t e s t)}$ for each segmented region are used for classification using $3 \sigma$ rule, $\mathrm{K}$ NN classifier, Neural Network classifier and Neuro Fuzzy classifier. The classification results are given in the Table 3 . The Fig. 4 shows some sample test images used for classification of spiral bacterial cells.

Table 2. Mean and standard deviation of geometric features of bacterial cells of types:

Vibrio, Spirillum and Spirochete

\begin{tabular}{|c|c|c|c|c|c|c|}
\hline \multirow{2}{*}{ Cell features } & \multicolumn{2}{|c|}{ Vibrio } & \multicolumn{2}{c|}{ Spirillum } & \multicolumn{2}{c|}{ Spirochete } \\
\cline { 2 - 7 } & $\begin{array}{c}\text { Mean } \\
\left(\bar{x}_{i}^{1}\right)\end{array}$ & $\begin{array}{c}\text { SD } \\
\left(\sigma_{i}^{1}\right)\end{array}$ & $\begin{array}{c}\text { Mean } \\
\left(\bar{x}_{i}^{2}\right)\end{array}$ & $\begin{array}{c}\text { SD } \\
\left(\sigma_{i}^{2}\right)\end{array}$ & $\begin{array}{c}\text { Mean } \\
\left(\bar{x}_{i}^{3}\right)\end{array}$ & $\begin{array}{c}\text { SD } \\
\left(\sigma_{i}^{3}\right)\end{array}$ \\
\hline Circularity $\left(\mathrm{x}_{1}\right)$ & 0.3550 & 0.0978 & 0.0928 & 0.0151 & 0.0612 & 0.0432 \\
\hline Eccentricity $\left(\mathrm{x}_{2}\right)$ & 0.9380 & 0.0433 & 0.9748 & 0.0054 & 0.9661 & 0.0388 \\
\hline Tortuosity $\left(\mathrm{x}_{3}\right)$ & 0.3665 & 0.0263 & 0.3871 & 0.0179 & 0.3917 & 0.0366 \\
\hline
\end{tabular}

Table 3. Classification accuracy for the different bacterial cells in the testing set images

\begin{tabular}{|c|c|c|c|c|c|c|c|}
\hline \multirow{2}{*}{$\begin{array}{c}\text { Spiral bacterial } \\
\text { cell types }\end{array}$} & \multirow{2}{*}{$\begin{array}{c}\text { No. of } \\
\text { cells in } \\
\text { test images }\end{array}$} & \multirow{2}{*}{$\begin{array}{c}3 \sigma \\
\text { classifier }\end{array}$} & \multicolumn{2}{|c|}{ K-NN classifier } & \multirow{2}{*}{$\begin{array}{c}\text { Neural } \\
\text { Network } \\
\text { classifier }\end{array}$} & $\begin{array}{c}\text { Neuro } \\
\text { Fuzzy } \\
\text { classifier }\end{array}$ \\
\cline { 4 - 8 } & & $\mathrm{K}=1$ & $\mathrm{~K}=3$ & $\mathrm{~K}=5$ & 100 & 100 \\
\hline Vibrio & 300 & 100 & 98.03 & 98.03 & 98.03 & 100 & 100 \\
\hline Spirillum & 840 & 100 & 93.54 & 93.54 & 93.54 & 100 \\
\hline Spirochete & 140 & 95 & 92.00 & 92.00 & 92.00 & 100 & 100 \\
\hline
\end{tabular}

The Table 3 summarizes the average classification rates obtained by different classification techniques. For testing images, the $3 \sigma$ classifier has yielded an accuracy of $95 \%$ to $100 \%$ and $\mathrm{K}-\mathrm{NN}$ classifier has yielded $92 \%$ to $98.03 \%$ for $\mathrm{K}=1$ (i.e. minimum distance classifier). The neural network classifier and the neuro fuzzy classifier have yielded 100\% accuracy. The performance comparison indicates that the neural network and neuro fuzzy classifier has good classification ability.
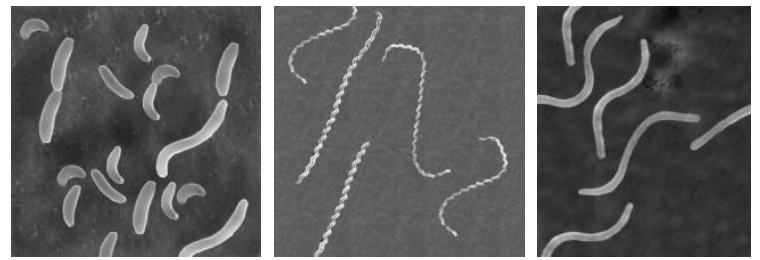

Fig. 4. Sample test images used for classification of spiral bacterial cells
Although the comparison of classification performance of the various state-of-the art methods in the literature is difficult because of the different cell image data sets used for experimentation, it may be observed that, in [1] statistical modeling techniques are applied for staphylococcus aureus cells and has yielded 98\%, in [3] data mining approach was used for HEp-2 cells and has yielded $86.67 \%$ classification rate, in [2] neural network approach has yielded above $90 \%$ classification rate in the various different types of bacterial cells and in [4] the statistical methodology has yielded classification rates in the range $89 \%$ to $98 \%$ for different categorization methods for fluorescent labeled cells. In [15] statistical analysis method for classification of various bacterioplankton groups was used and has yielded $80 \%$ overall accuracy. The proposed method is computationally less expensive and yet yields comparable classification rates. The $3 \sigma$ classifier has yielded an accuracy in the range of $95 \%$ to $100 \%$ and K-NN classifier has yielded $92 \%$ to $98.03 \%$ for $\mathrm{K}=1$ (i.e. minimum distance classifier). The neural network 
classifier and the neuro fuzzy classifier have yielded $100 \%$ accuracy for different spiral bacterial cell groups.

\section{CONCLUSIONS}

In this paper, we have proposed automated cell identification and classification by segmenting digital microscopic spiral bacterial cell images using active contour method and extracting geometric features of cells. The experimental results are compared with the manual results obtained by expert. The proposed method is computationally less expensive and yet yields comparable classification rates. The $3 \sigma$ classifier has yielded an accuracy in the range of $95 \%$ to $100 \%$ and K-NN classifier has yielded $92 \%$ to $98.03 \%$ for $\mathrm{K}=1$ (i.e. minimum distance classifier). The neural network classifier and the neuro fuzzy classifier have yielded 100\% accuracy for different spiral bacterial cell types.

\section{ACKNOWLEDGEMENTS}

The authors are grateful to the referees for their valuable comments and suggestions. Further, the authors are also indebted to Dr. A. Dayanand, Professor of Microbiology, Gulbarga University, Gulbarga and Dr. Ramakrishna, Department of Microbiology, Government Degree College, Gulbarga, for providing bacterial cell images and manual results of the cell images by visual inspection. This research work is financially supported under UGC Minor Research Project (F. No. MRP(S)-715/2010-11/KAGU009/UGCSWRO).

\section{REFERENCES}

[1] Aneja, K. R. (2002). Experiments in Microbiology Plant Pathology Tissue Culture and Mushroom Culture, Newage International Publications, New Delhi, India.

[2] Carolina, W., Joakim, L., Mikael, V., Ewert, B. and Lennart, B., Algorithms for Cytoplasm Segmentation of Fluorescence Labeled Cells, Analytical Cellular Pathology, Vol.24, 2002, pp.101-111.

[3] Obtained through the Internet www.cellbank.nibio.go, http://denniskunkel.com/DK/Bacteria/en.academic.ru/dic .nsf/enwiki/76356,

[4] Hiremath P. S. and Parashuram Bannigidad, Automatic Identification and Classification of Cocci Bacterial Cells using Digital Microscopic Images, Int'l. J. on computational Biology and Drug Design (IJCBDD), Inderscience Publishers Ltd. USA, Vol. 4, No. 3, pp. 262-273, 2011.

[5] Tony F Chan, Luminita A. Vese, Active Contours Without Edges, IEEE Transaction on Image Processing, Vol. 10, No. 2, February 2001.

[6] Michael Kass, Andrew witkin and demetri, Snakes : Active contour models, Intl. J. of Computer Vision, 1998, pp. 321-331.

[7] Liu, J. F.B. Dazzo, O. Glagovela, B. Yu, A.K. Jain, CMEIAS: A Computer-Aided System for the Image Analysis of Bacterial Morphotypes in Microbial Communities, Springer-Verlag, Microb. Ecol. Vol.41, 2001, pp. 173-194.
[8] Jeffrey C. Pommerville (2010) Alcamo's Fundamentals of Microbiology Body Systems Edition, Jones and Bartlett Publishers, USA.

[9] Nicholas, B., Åke, H., Johan, W., Rocio, C-H. and Peter, K.B., Rapid Determination of Bacterial Abundance, Biovolume, Morphology, and Growth by Neural Network-Based Image analysis, Applied and Environmental Microbiology, Vol.64(9), 1998, pp.32463255 .

[10] Pattan Prakash C., V.D. Mytri and P.S. Hiremath. (2010) Classification of Cast Iron Based Graphite Grain Morphology using Neural Network Approach, $2^{\text {nd }}$ International Conference on Digital Image Processing (ICDIP-2010), Proc. of SPIE Vol. 7546-53, Feb. 26-28, 2010, Singapore, pp. 75462S-1-6.

[11] Petra Perner, Classification of HEp-2 Cells using Fluorescent Image Analysis and Data Mining, Medical Data Analysis, Springer Verlag, LNCS Vol.2199, 2001, pp.219-224.

[12] Rafael C. Gonzalez and Richard E. Woods (2002). Digital Image Processing, Pearson Education Asia, India.

[13] Sigal Trattner and Greenspan H, Automatic Identification of Bacterial Types Using Statistical Imaging Methods, IEEE Transactions on Medical Imaging, Vol.23(7), 2004, pp.807-820.

[14] Thomas, P., Josef, F., Martin, P. and Michaela, M.S. (2009) New Image Analysis Tool to Study Biomass and Morphotypes of Three Major Bacterioplankton Groups in an Alpine Lake, Acuatic Microbiol Ecology, Vol.54: pp. 113-126.

[15] Venkataraman, S., Allison, D.P., Qi, H., Morrell-Falvey, J.L., Kallewaard, N.L., Crowe Jr., J.E.and Doktycz, M.J. (2006) Automated Image Analysis of Atomic Microscopy Images of Rotavirus Particles, Ultramicroscopy, Elsevier, Vol. 106, 2006, pp. 829-837.

[16] Pekka Ruusuvuori, Jenni Seppaal, Timo Erkkil, Antti Lehmussola, Jaakko A. Puhakka and Olli Yli-Harja, Efficient Automated Method for Image-based Classification of Microbial Cells, Proc. of the IEEE 19th Int'l. Conf. on Pattern Recognition, (ICPR 2008), Tampa, Florida, USA, 7-11 December 2008, pp. 1-4.

[17] John C. Russ, The Image Processing Hand Book, (2007), $5^{\text {th }}$ Ed. CRC Press, New Jersey.

[18] Evangelia Micheli-Tzanakou (2000) Supervised and Unsupervised Pattern Recognition - Feature Extraction and Computational Intelligence, CRC Press LLC, Florida.

[19] Hiremath P.S. and Parashuram Bannigidad, Digital Microscopic Image Analysis of Spiral Bacterial Cell Groups, Int'l. Conf. on Intelligent Systems and Data Processing (ICISD-2011), Gujarat, Jan. 24-25 ${ }^{\text {th }}, 2011$, pp. 209-213.

[20] Chenyang Xu, Jerry L Prince, Snakes , Shapes, and Gradient Vector Flow, IEEE Transactions on Image Processing, Vol. 7, No. 3, March 1998. 